\title{
Recurrent Laryngeal Nerve Paralysis after Esophagectomy: Respiratory Complications and Role of Nerve Reconstruction
}

\author{
Kazuo Koyanagi, ${ }^{1}$ Hiroyasu Igaki, ${ }^{1}$ Jun Iwabu, ${ }^{1}$ Hiroki Ochiai ${ }^{2}$ and \\ Yuji Tachimori $^{1}$ \\ ${ }^{1}$ Division of Esophageal Surgery, Department of Gastrointestinal Oncology, National Cancer Center Hospital, \\ Tokyo, Japan \\ ${ }^{2}$ Division of Colorectal Surgery, Department of Gastrointestinal Oncology, National Cancer Center Hospital, \\ Tokyo, Japan
}

\begin{abstract}
Recurrent laryngeal nerve paralysis (RLNP) after esophagectomy is a common complication and associated with aspiration pneumonia. In this study, we assessed the risk of RLNP and the usefulness of immediate reconstruction of recurrent laryngeal nerve (RLN) to prevent respiratory complications after esophagectomy. Seven hundred and eighty-two consecutive patients underwent an esophagectomy with three-field lymph node dissection, simultaneous gastric conduit reconstruction, and cervical anastomosis. Vocal cord function was observed using a flexible laryngoscope. Reconstruction between RLN and ipsilateral vagus nerve was performed during esophagectomy. RLNP was observed in $229(29.3 \%)$ of the patients after esophagectomy: 198 unilateral and 31 bilateral cases. Of the 198 unilateral RLNP, vocal cord paralysis was observed predominantly on the left side (82.7\%). RLNP was significantly associated with postoperative respiratory complications $(P<0.001)$ requiring a tracheotomy $(P<0.001)$ and mechanical ventilation $(P<0.001)$ and was also associated with esophagogastric anastomotic leakage $(P=0.015)$; consequently, the postoperative hospital stay was longer for patients with RLNP $(P<0.001)$. A longer operation time $(P<0.001)$ and advanced age $(P=0.038)$ were identified as significant independent predictors of RLNP. Resection of the RLN together with metastatic nodes was performed in 29 cases. The patients underwent RLN reconstruction $(n=11)$ had a significantly shorter postoperative hospital stay than those without RLN reconstruction $(\mathrm{n}=18)(P=0.019)$. In conclusion, RLNP was related to a poorer postoperative course among patients undergoing an esophagectomy. New surgical technologies are recommended for prevention of RLNP.
\end{abstract}

Keywords: esophageal cancer; esophagectomy; nerve reconstruction; recurrent laryngeal nerve paralysis; respiratory complication

Tohoku J. Exp. Med., 2015 September, 237 (1), 1-8. C 2015 Tohoku University Medical Press

\section{Introduction}

The incidence of esophageal cancer has been increasing over the past two decades (Parkin et al. 1999; Jemal et al. 2011). Esophagectomy is the mainstay of curative modalities for localized esophageal cancer. A transthoracic esophagectomy with cervical, mediastinal, and abdominal (three-field) lymph node dissection has been performed as a standard surgical procedure for thoracic esophageal cancer in Japan (Igaki et al. 2004; Fujita 2013). However, this procedure is relatively invasive and requires a complex operation. Therefore, morbidity is a major concern during the follow-up period.

Recurrent laryngeal nerve paralysis (RLNP) is a common and sometimes severe complication after esophagectomy. The dissection of cervical paraesophageal and tho- racic paratracheal lymph nodes, especially along the recurrent laryngeal nerve (RLN), raises the risk of injury to the RLN. The incidence of RLNP after esophagectomy has been reported to range from $0 \%$ to $50 \%$ (Pertl et al. 2011). This wide range is related to several factors, including the extent of lymph node dissection, the site of anastomosis, and the method of postoperative laryngeal examination (Walther et al. 2003; Jeannon et al. 2009). RLNP may occur unilaterally or bilaterally and is predominantly expressed as hoarseness and aphonia. Although most unilateral RLNP (URLNP) is transient and hoarseness is not itself troublesome, patients suffering from RLNP sometimes develop secondary respiratory complications, such as aspiration pneumonia and atelectasis; consequently, the duration of unexpected intubation and the patient's stay in the intensive care unit may be prolonged. Therefore, efforts

Received June 15, 2015; revised and accepted July 21, 2015. Published online August 11, 2015; doi: 10.1620/tjem.237.1.

Correspondence: Kazuo Koyanagi, M.D., Ph.D., Division of Esophageal Surgery, Department of Gastrointestinal Oncology, National

Cancer Center Hospital, 5-1-1 Tsukiji, Chuo-ku, Tokyo 104-0045, Japan.

e-mail: kkoyanag@ncc.go.jp 
to prevent RLN damage as well as to assess RLNP promptly are essential for the postoperative management of patients undergoing an esophagectomy. The generally accepted management methods for patients with URLNP are observation for several months after surgery, intracordal injection (Graboyes et al. 2011), type I thyroplasty (Isshiki et al. 1989; Schneider et al. 2003), arytenoids adduction (Isshiki et al. 1978), and laryngeal reinnervation. In cases with RLN involvement via metastatic nodes, the RLN is sometimes resected together with metastatic nodes to enable a curative operation (Tachimori et al. 1995). Laryngeal reinnervation should be considered in such cases, since this procedure can prevent the progressive loss of thyroarytenoid muscle tone and stiffness, which cannot be restored reliably using other techniques (Lu et al. 1996; McCulloch et al. 2002).

In this study, we retrospectively reviewed our recent experience with RLNP occurring after an esophagectomy in patients with esophageal cancer to clarify the risk of RLNP and its association with respiratory complications. Additionally, we focused on the immediate reconstruction of the RLN during esophagectomy as a means of preventing respiratory complications.

\section{Materials and Methods}

\section{Patients}

Between January 2005 and October 2014, a total of 1,352 esophageal cancer patients underwent an esophagectomy at our institute. Among them, 782 patients who received the same surgical procedures (resection of the esophagus with three-field lymph node dissection, simultaneous reconstruction using a gastric conduit, and anastomosis at the neck) were enrolled in the study.

The 782 patients comprised 657 males and 125 females with a median age of 64 years (range, 30-80 years). Of them, 732 (93.6\%), $25(3.2 \%)$, and $25(3.2 \%)$ patients histopathologically presented with squamous cell carcinoma, adenocarcinoma, and other cancer types, respectively. Almost all the tumors (96.5\%) were located at the thoracic esophagus: 99,375 , and 280 patients had tumors in the upper, middle, and lower third of the thoracic esophagus, respectively. The cancer stage was determined according to the 7th edition of TNM Classification of Malignant Tumors (International Union Against Cancer 2009). There were 207, 67, 475, and five patients categorized as T1, T2, T3, and T4 tumors, respectively. Also, there were 187, 369,184 , and 15 patients categorized as N0, N1, N2, and N3 lymph node metastasis, respectively. Based on the diagnosis of these categories, the patients were clinically classified as IA $(n=88)$, IB $(n=26)$, IIA $(\mathrm{n}=81)$, IIB $(\mathrm{n}=146)$, IIIA $(\mathrm{n}=225)$, IIIB $(\mathrm{n}=144)$, IIIC $(\mathrm{n}=$ $14)$, and IV $(n=58)$. All the cStage IV patients had supraclavicular lymph node metastasis, but none of them had organ metastasis. Preoperative therapy was performed in 375 (48.0\%) patients: chemotherapy and chemoradiotherapy were performed in 316 and 59 patients, respectively.

Assessment of laryngopharyngeal function, respiratory complications, and anastomotic leakage

During the preoperative examinations, the oropharynx, hypopharynx, and larynx were evaluated using esophagogastric endoscopy to determine whether any diseases or dysfunction existed. Six

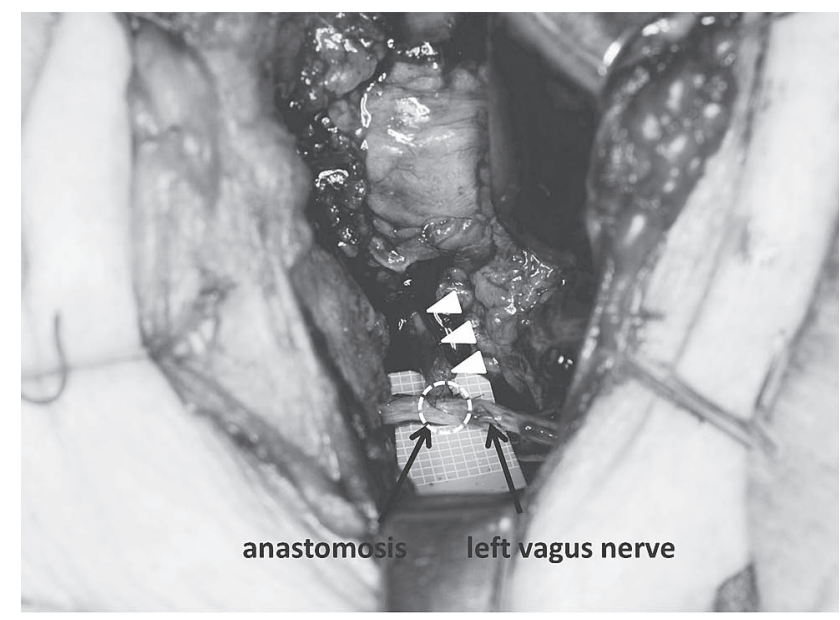

Fig. 1. The end-to-side anastomosis between left RLN and ipsilateral vagus nerve was performed under a microscope. Arrowheads indicate left RLN. The anastomotic site is shown within dotted circle.

patients exhibited preoperative URLNP: left-side paralysis was present in five patients, and right-side paralysis was present in one patient. However, none of the patients exhibited aspiration pneumonia or swallowing dysfunction preoperatively. At our institute, mechanical respiratory support after surgery was not routinely used, and extubation of the endotracheal tube was performed in the operating room. All the patients were examined using a flexible laryngoscope on the first postoperative day and as needed thereafter to check the movement of the vocal cord. Aspiration was assessed using videofluorography. Respiratory complications included the pneumonia, atelectasis, empyema, and mediastinal abscess. Anastomotic leakage was diagnosed by clinical findings and postoperative esophagography.

\section{Reconstruction of RLN}

Immediate RLN reconstruction was performed at the time of esophageal cancer surgery. We used the ipsilateral vagus nerve for reinnervation. After trimming the edge of RLN and mobilization of vagus nerve, end-to-side neurorrhaphy using six stitches with interrupted sutures of 8-0 polypropylens was performed under a microscope by plastic surgeons (Fig. 1). When the reconstruction of the RLN was performed on the left side of the neck, the gastric conduit was elevated via a posterior mediastinal route to avoid any stress to the anastomosis of the nerve.

\section{Statistical analysis}

A chi-square test and an exact t-test were used to assess the associations between the patient characteristics and the incidence of RLNP as well as between surgical outcomes (operation time, blood loss, morbidity, and mortality) and RLNP. The Wilcoxon signed-rank test was used to assess the association between postoperative hospital stay and RLNP. For analyses among patients with RLN resection, similar statistical methods were used. Univariate analyses of the risk of RLNP according to clinicopathological factors were examined using the log-rank test. Both known clinical and pathological risk factors for postoperative outcomes such as $\mathrm{cT}$ or cN category, TNM staging, and preoperative therapy, and individual risk factors selected by univariate analyses were included in the model. A multinomial logistic regression analysis was used for predictive variable selection 
of the occurrence of RLNP. The survival curves were generated using the Kaplan-Meier method. To examine overall survival according to clinical factors, the log-rank test was used. The analysis was performed using JMP software (version 12; SAS Institute, Cary, NC, USA) and all the tests were two-sided with a significance level $\leq 0.05$.

\section{Results}

RLNP was observed in 229 (29.3\%) of the 782 patients after esophagectomy. There were 198 unilateral cases and 31 bilateral cases of paralysis. Among the patients with URLNP, $158(82.7 \%)$ patients and $40(17.3 \%)$ patients had paralysis on the left and right sides of the vocal cord, respectively. Resection of the RLN along with the tumor was performed in 29 patients: on the left side in 18 patients and on the right side in 11 patients. Between 2005 and 2009, 435 patients underwent surgery, and RLNP was observed in 125 (28.7\%) patients. Between 2010 and 2014, 347 patients underwent surgery, and RLNP was observed in $104(30.0 \%)$ patients. No significant difference was observed between the earlier and later periods $(P=0.71)$.

\section{Patients' characteristics}

We divided the patients into two groups: those with and those without RLNP after esophagectomy (RLNP (+) and RLNP $(-)$, respectively). The mean ages of the patients were $64.3 \pm 8.5$ years in the RLNP $(+)$ group and $63.4 \pm 7.8$ years in the RLNP (-) group, however, the difference was not significant. Gender, histopathology, tumor location, cT or $\mathrm{cN}$ category, TNM staging, and preoperative therapy did not differ significantly between the two groups (data not shown). Also, the incidence of metastasis to the lymph node along the RLN (cervical paraesophageal and thoracic paratracheal) in the RLNP (+) group was not significantly different from that in the RLNP $(-)$ group $(21.0 \%$ vs. $25.5 \%, P=0.18$ ).

\section{Comparison of surgical outcome according to RLNP}

The mean operative time was significantly longer in the RLNP $(+)$ group than in the RLNP $(-)$ group $(P=$ $0.001)$, whereas no significant difference in blood loss was observed between the two groups (Table 1). RLNP was associated with other postoperative complications, such as respiratory complications $(P<0.001)$ and esophagogastric anastomotic leakage $(P=0.015)$. The incidence of tracheotomy $(P<0.001)$ and unexpected mechanical ventilation $(P<0.001)$ because of respiratory complications was higher in the RLNP (+) group than in the RLNP (-) group. Consequently, the postoperative hospital stay was significantly longer for patients with RLNP than for those without RLNP $(P<0.001)$. The hospital mortality rate was not related to the occurrence of RLNP.

\section{Risk assessment for RLNP}

In subsequent analyses, parameters such as age, operative time, and blood loss were divided into two groups according to the median value. As the median overall age was 64 years, the patients were divided into two groups: those with an age of 64 years or more $(n=369)$, and those with an age of 63 years or less $(n=413)$. Similarly, the patients were divided into two groups according to those with an operative time of $438 \mathrm{~min}$ or more $(\mathrm{n}=390)$ and those with an operative time of 437 min or less $(n=392)$, as well as those with a blood loss of $381 \mathrm{~mL}$ or more $(\mathrm{n}=$ 391) and those with a blood loss of $380 \mathrm{~mL}$ or less ( $\mathrm{n}=$ 391). Univariate analyses using the log-rank test showed that an age of over 64 years $(P=0.028)$, an operation time of $438 \mathrm{~min}$ or more $(P=0.004)$, and the use of a thoracoscopic esophagectomy $(P=0.045)$ were significantly correlated with the occurrence of RLNP after esophagectomy (Table 2). Gender, histopathology (squamous cell carcinoma vs. others), tumor location (upper vs. middle vs. lower esophagus), cT category ( $1 / 2$ vs. $3 / 4), \mathrm{cN}$ category (0/1 vs. $2 / 3)$, cStage (I/II vs. III/IV), preoperative therapy, lymph node metastasis along the RLN, and blood loss (381 $\mathrm{mL}$ or more vs. $380 \mathrm{~mL}$ or less) were not correlated with the occurrence of RLNP. Based on the results of these univariate analyses, we conducted a multivariate analysis using a logistic regression analysis to assess the risk factors for RLNP after an esophagectomy; a longer operation time (odds ratio, 1.70; 95\% CI, 1.25-2.33; $P<0.001$ ) and an

Table 1. Comparison of surgical outcomes between patients with RLNP and patients without RLNP after esophagectomy.

\begin{tabular}{lccc}
\hline & $\begin{array}{c}\text { RLNP }(+) \\
(\mathrm{n}=229)\end{array}$ & $\begin{array}{c}\text { RLNP }(-) \\
(\mathrm{n}=553)\end{array}$ & $P$ value \\
\hline Operation time (min; mean $\pm \mathrm{SD})$ & $466.5 \pm 116.7$ & $438.9 \pm 99.7$ & 0.001 \\
Blood loss (mL; mean $\pm \mathrm{SD})$ & $504.2 \pm 600.2$ & $463 \pm 331$ & 0.23 \\
Morbidity & & & $<0.001$ \\
$\quad$ Respiratory complications & $84(36.7 \%)$ & $104(18.8 \%)$ & 0.015 \\
$\quad$ Anastomotic leakage & $50(21.8 \%)$ & $80(14.5 \%)$ & $<0.001$ \\
Tracheotomy & $13(5.7 \%)$ & $8(1.4 \%)$ & $<0.001$ \\
Mechanical ventilation & $24(10.5 \%)$ & $22(4.0 \%)$ & $<0.001$ \\
Postope. hospital stay (day; mean $\pm \mathrm{SD})$ & $34.1 \pm 36.6$ & $23.1 \pm 25.0$ & 0.26 \\
Hospital mortality & $6(2.6 \%)$ & $8(1.4 \%)$ & \\
\hline
\end{tabular}

RLNP, recurrent laryngeal nerve paralysis. 
Table 2. Univariate analyses of clinical factors for prediction of RLNP after esophagectomy.

\begin{tabular}{|c|c|c|c|c|}
\hline \multicolumn{2}{|l|}{ Factors } & \multirow{2}{*}{$\frac{\text { Patients (n) }}{369}$} & \multirow{2}{*}{$\frac{\text { No. of RLNP }(\%)}{122(33.1)}$} & \multirow{2}{*}{$\frac{\text { Log-rank test }(P)}{0.028}$} \\
\hline Age (years) & $\geq 64$ & & & \\
\hline & $<64$ & 413 & 107 (25.9) & \\
\hline \multirow[t]{2}{*}{ Gender } & Male & 657 & $182(27.7)$ & 0.12 \\
\hline & Female & 125 & $47(37.6)$ & \\
\hline \multirow[t]{2}{*}{ Histopathology } & $\mathrm{Sq}$ & 732 & $209(28.6)$ & 0.17 \\
\hline & Non-sq & 50 & $20(40)$ & \\
\hline \multirow[t]{3}{*}{ Tumor location } & Upper & 99 & $26(26.2)$ & 0.81 \\
\hline & Middle & 375 & $109(29.1)$ & \\
\hline & Lower & 280 & $83(29.4)$ & \\
\hline \multirow[t]{2}{*}{ cT category } & $\mathrm{cT} 1 / 2$ & 276 & $77(27.9)$ & 0.61 \\
\hline & $\mathrm{cT} 3 / 4$ & 479 & $142(29.6)$ & \\
\hline \multirow[t]{2}{*}{$\mathrm{cN}$ category } & $\mathrm{cN} 0 / 1$ & 566 & $172(30.3)$ & 0.64 \\
\hline & $\mathrm{cN} 2 / 3$ & 199 & $57(28.7)$ & \\
\hline \multirow[t]{2}{*}{ cStage } & $\mathrm{I} / \mathrm{II}$ & 317 & $99(31.2)$ & 0.33 \\
\hline & III/IV & 437 & $121(27.7)$ & \\
\hline \multirow[t]{2}{*}{ Preoperative therapy } & yes & 374 & $115(30.7)$ & 0.19 \\
\hline & no & 395 & $106(26.8)$ & \\
\hline \multirow[t]{2}{*}{ LN metastasis along RLN } & Positive & 189 & $48(25.4)$ & 0.13 \\
\hline & Negative & 593 & $181(30.4)$ & \\
\hline \multirow[t]{2}{*}{ Operation time (min) } & $\geq 438$ & 390 & $136(34.9)$ & 0.004 \\
\hline & $<438$ & 392 & $93(23.7)$ & \\
\hline \multirow[t]{2}{*}{ Blood loss (mL) } & $\geq 381$ & 391 & $115(29.4)$ & 0.17 \\
\hline & $<381$ & 391 & $114(29.2)$ & \\
\hline \multirow[t]{2}{*}{ Thoracoscopic esophagectomy } & yes & 169 & $58(34.3)$ & 0.045 \\
\hline & no & 613 & $171(27.9)$ & \\
\hline
\end{tabular}

RLNP, recurrent laryngeal nerve paralysis; Sq, squamous cell carcinoma; LN, lymph node; RLN, recurrent laryngeal nerve.

Table 3. Multivariate analysis of clinical factors for prediction of RLNP after esophagectomy.

\begin{tabular}{lccr}
\hline \multicolumn{1}{c}{ Factors } & odds ratio & $95 \%$ CI & $P$ \\
\hline Operation time $(\mathrm{min} ; \geq 438$ vs. $<438)$ & 1.70 & $1.25-2.33$ & $<0.001$ \\
Age $($ years; $\geq 64$ vs. $<64)$ & 1.38 & $1.01-1.90$ & 0.038 \\
\hline
\end{tabular}

RLNP, recurrent laryngeal nerve paralysis.

elderly age (odds ratio, $1.38 ; 95 \% \mathrm{CI}, 1.04-1.90 ; P=0.038$ ) were selected as independent significant predictors of RLNP after an esophagectomy (Table 3). A survival curve based on the occurrence of RLNP after esophagectomy was generated using the Kaplan-Meier methods (Fig. 2). The estimated five-year overall survival rate was $61.1 \% \pm 4.3 \%$ for the RLNP (+) group and $69.7 \% \pm 2.3 \%$ for the RLNP $(-)$ group. No difference in overall survival was observed between patients with and those without $\operatorname{RLNP}(P=0.25)$.

\section{Association between reconstruction of $R L N$ and surgical outcome}

Of the 29 patients whose RLN was resected together with metastatic lymph nodes, reconstruction of the RLN using the ipsilateral vagus nerve was performed in 11 patients (left side in 8 patients and right side in 3 patients).
Postoperative respiratory complications occurred in 3 (27.3\%) of the 11 patients with RLN reconstruction and 9 $(50 \%)$ of the 18 patients without RLN reconstruction (Table 4). However, this difference was not significant. Similarly, the incidences of tracheotomy, mechanical ventilation, and hospital mortality tended to be higher among patients without RLN reconstruction, compared with those with RLN reconstruction, although the differences were not significant. The postoperative hospital stay was significantly longer among patients without RLN reconstruction than in those with RLN reconstruction $(P=0.019)$. Although the backgrounds of the patients were not identical, we assessed the overall survival according to RLN reconstruction (Fig. 3). The estimated three-year overall survival rate for patients with RLN reconstruction $(72.0 \% \pm 17.8 \%)$ was better than that for patients without RLN reconstruction 


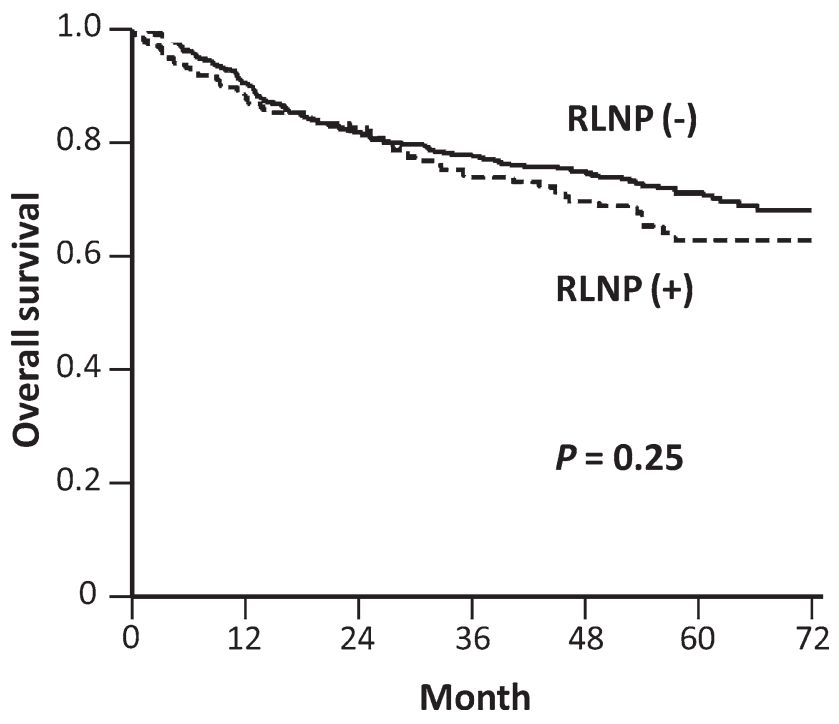

Fig. 2. Kaplan-Meier survival curve based on the RLNP after esophagectomy.

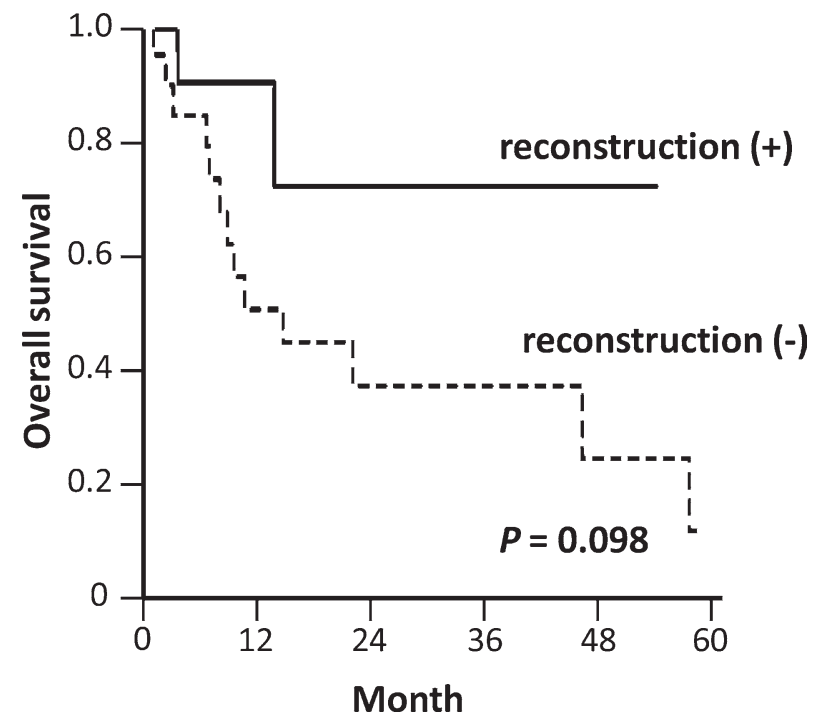

Fig. 3. Kaplan-Meier survival curve based on the reconstruction of the RLN.

Table 4. Comparison of surgical outcomes between patients with reconstruction of RLN and patients without reconstruction of RLN.

\begin{tabular}{lccc}
\hline & $\begin{array}{c}\text { Reconstruction }(+) \\
(\mathrm{n}=11)\end{array}$ & $\begin{array}{c}\text { Reconstruction (-) } \\
(\mathrm{n}=18)\end{array}$ & $P$ value \\
\hline Respiratory complications (\%) & $3(27.3)$ & $9(50)$ & 0.23 \\
Tracheotomy (\%) & $0(0)$ & $4(22.2)$ & 0.092 \\
Mechanical ventilation (\%) & $0(0)$ & $3(16.7)$ & 0.14 \\
Postope. hospital stay (day; mean \pm SD) & $28.4 \pm 18.3$ & $37.7 \pm 31.7$ & 0.019 \\
Hospital mortality (\%) & $0(0)$ & $2(11.1)$ & 0.25 \\
\hline
\end{tabular}

RLN, recurrent laryngeal nerve.

$(37.6 \% \pm 11.9 \%)$; however, the difference was not significant $(P=0.098)$.

\section{Discussion}

The present study demonstrated that RLNP was significantly associated with respiratory complications after esophagectomy and might also prolong the postoperative hospital stay. Because a longer operation time and an elderly age enhanced the risk of RLNP, surgeons should pay greater attention not to injure the RLN during lymph node dissection along the nerve. The immediate reconstruction of the RLN during an esophagectomy may not only prevent secondary respiratory complications, but it is also likely to maintain the patient's quality of life.

The recurrent laryngeal nerves run in the tracheoesophageal grooves on both sides; the right recurrent nerve turns around the subclavian artery and runs cranially, and the left recurrent nerve turns around the aortic arch and runs up through the thoracic cavity before entering the cervical fields. The cervical esophagus is usually pulled upwards from the left side of the neck and the esophagogastric anastomosis is performed. Injury to the RLN is thought to result from damage caused by the electrocautery device, stretching, or compression of the nerve during surgery and postoperative edema or hematoma. The left RLN has an increased risk of injury, compared with the right RLN, because of its longer length. In fact, vocal cord paralysis was predominantly observed on the left side in the study. Other studies have reported relatively lower incidences of RLNP (Walther et al. 2003; Gockel et al. 2005). However, the incidence may partly depend on the diagnostic methods (Jeannon et al. 2009). When clinical findings or indirect laryngoscopy findings are selected as the diagnostic method, the sensitivity of RLNP diagnosis may decrease. The direct observation of vocal cord motility through a laryngoscope offers a more detailed evaluation of the larynx; consequently the incidence of RLNP likely to increases. An accurate diagnosis of RLNP is needed for the appropriate management of patients after an esophagectomy.

Previous reports have suggested that suprabifurcational lymph node dissection and cervical esophagogastric anastomosis may increase the incidence of RLNP (Hulscher et al. 1999; Walther et al. 2003). An Ivor-Lewis esophagectomy, in which cervical and upper mediastinal lymph node 
dissection is not performed and the esophagogastric anastomosis is located in the intrathoracic cavity, is considered to be useful for avoiding RLNP. However, this method is only used for lower thoracic esophageal cancer. In Japan, a transthoracic esophagectomy with thorough lymph node dissection after neoadjuvant chemotherapy is a standard treatment method for localized esophageal cancer (Ando et al. 2012). Our results may support this surgical strategy, since no differences in RLNP were observed according to tumor location and lymph node metastases along the RLN. Rigid and reliable surgical procedures allow radical surgery to be performed in a manner that is not dependent on the tumor status.

Because the RLN is a major motor nerve of the larynx and also gives off branches to the cricopharyngeus muscles which form the upper esophageal sphincter and play a pivotal role in swallowing, RLNP is closely related to impairments in breathing, speaking, coughing, and swallowing. In patients with RLNP, both the incidence and the severity of aspiration pneumonia can be increased as a result of reflux caused by the delayed emptying of the gastric conduit, especially during the early postoperative period (Perie et al. 1998). The study demonstrated an obvious association between RLNP and postoperative respiratory complications that frequently required intensive care management, such as a tracheotomy and reintubation. In cases with bilateral RLNP, the bilateral vocal cords assume a median position, often leading to asphyxia and necessitating urgent reintubation or tracheotomy. In the present study, the incidence of esophagogastric anastomotic leakage was associated with RLNP, especially on the left side $(21.7 \%$ on left side and $11.6 \%$ on right side). The reason for this result is uncertain. However, severe infection of the neck after anastomotic leakage might be related to vocal cord dysfunction, since RLNP occurred not only as a result of direct surgical damage to the nerve, but also because of postoperative edema or hematoma around the nerve. Postoperative respiratory complications, anastomotic leakage, and RLNP are considered to be major complications after an esophagectomy. In the present study, RLNP was associated with postoperative respiratory complications and anastomotic leakage as well as a longer postoperative hospital stays. Thus, proper precautions against RLNP can improve the management of patients after an esophagectomy.

Intimate knowledge of normal and anatomic variants of the RLN is very important for avoiding injury to the RLN (Tateda et al. 2008). Recently, new techniques have been introduced in esophageal cancer surgery. Intraoperative RLN monitoring is widely used in thyroid and parathyroid surgery. This technique, which is based on an electrically stimulated response, has recently been adopted for intrathoracic surgeries, and several investigators have demonstrated the technical feasibility and clinical benefits for reducing the incidence of RLNP and postoperative pneumonia among patients undergoing transthoracic esophagectomy (Gelpke et al. 2010; Zhong et al. 2014). Surgeons usually use electrocautery for hemostasis. However, electrocautery can deliver a high temperature of between $100^{\circ} \mathrm{C}$ and $1,200^{\circ} \mathrm{C}$, and these extremely high temperatures can injure the RLN. Rino et al. (2014) reported that the use of absorbable dense knit hemostat, instead of an electrocautery device, for hemostasis around the RLN could prevent the RLNP during esophageal cancer surgery. Thoracoscopic esophagectomy has been employed as minimally invasive surgery (Saikawa et al. 2014; Koyanagi et al. 2015). Although magnification of the surgical fields can enable a precise lymph node dissection along the RLN, the advantages of a thoracoscopic esophagectomy for reducing the RLNP continues to be debated.

An upper thoracic tumor location, suprabifurcational mediastinal lymph node dissection, and cervical anastomosis may increase the incidence of RLNP after esophagectomy. However, to date, no studies have elucidated the risk of RLNP among patients undergoing an esophagectomy with three-field lymph node dissection and cervical esophagogastric anastomosis. In the present study, all the patients underwent such surgical procedures and a laryngoscope examination of the vocal cord after esophagectomy, and neither the tumor location nor lymph node metastasis along the RLN was selected as a risk factor for RLNP, whereas a longer operation time and an elderly age significantly increased the risk of RLNP. The operation time is usually associated with the extent of tumor progression, and surgeons may become fatigued as the operation time lengthens. The strength of tissues, possibly including nerves, in elderly patients is thought to be lower than that of younger patients (Iida et al. 2013). These results demonstrate that gentle and precise surgical maneuvers are absolutely essential to prevent RLNP.

Previous reports have demonstrated that RLN reconstruction can improve phonation in patients with thyroid cancer (Miyauchi et al. 2009; Chhetri and Blumin 2012). The RLN consists of adductor and abductor fibers that innervate adductor and abductor muscles, respectively. There is no spatial segregation of the nerve fibers. After the direct anastomosis of the served RLN, the nerve fibers regenerate in a misdirected fashion. However, there are two to four times as many adductors as abductor fibers in the RLN; consequently, the reinnervated vocal cords are usually fixed at the median and the laryngeal muscle tone and mass can be restored. Our results in patients with esophageal cancer were similar to those of patients with thyroid cancer. In the present study, the incidence of postoperative respiratory complications, tracheotomy, and mechanical ventilation decreased among patients who underwent RLN reconstruction. Although we did not assess the phonation time or glottal gap, the lower incidence of aspiration after esophagectomy suggested the maintenance of laryngeal function. Patients with severe RLNP may suffer from decreased respiratory function for a long period after surgery, and their quality of life may deteriorate. Aspiration pneumonia can be a life-threatening condition, 
especially among elderly patients. A tendency toward a better survival outcome was observed for patients with RLN construction, compared with that for patients without RLN, although the difference was not significant. This result supports the idea that laryngeal function should be maintained or restored by additional procedures to prevent aspiration pneumonia in patients with severe RLNP. Among the numerous management methods available for RLNP, including intracordal injection, type I thyroplasty, and arytenoids adduction, reconstruction of the RLN during esophagectomy should be considered, since this procedure can reliably restore the thyroarytenoid muscle tone and stiffness, compared with other techniques.

Although this study analyzed patients retrospectively, we assessed a consecutive and large series of esophageal cancer patients who received similar surgical procedures at one institute. The association between RLNP and a poorer surgical outcome was clearly elucidated in the present series of patients with esophageal cancer. Reconstruction of the RLN using the ipsilateral vagus nerve can improve postoperative management after an esophagectomy. Because the maintenance of quality of life after an esophagectomy probably contributes to the long-term survival outcome, further studies examining surgical technologies that may reduce or prevent RLNP should be considered.

\section{Conflict of Interest}

The authors declare no conflict of interest.

\section{References}

Ando, N., Kato, H., Igaki, H., Shinoda, M., Ozawa, S., Shimizu, H., Nakamura, T., Yabusaki, H., Aoyama, N., Kurita, A., Ikeda, K., Kanda, T., Tsujinaka, T., Nakamura, K. \& Fukuda, H. (2012) A randomized trial comparing postoperative adjuvant chemotherapy with cisplatin and 5-fluorouracil versus preoperative chemotherapy for localized advanced squamous cell carcinoma of the thoracic esophagus. Ann. Surg. Oncol., 19, 68-74.

Chhetri, D.K. \& Blumin, J.H. (2012) Laryngeal reinnervation for unilateral vocal fold paralysis using ansa cervicalis nerve to recurrent laryngeal nerve anastomosis. Ope. Techniq. Otolaryngol., 23, 173-177.

Fujita, H. (2013) President's address of the 65th annual scientific meeting of the Japanese Association for Thoracic Surgery: challenges for advanced esophageal cancer. Gen. Thorac. Cardiovasc. Surg., 61, 201-207.

Gelpke, H., Grieder, F., Decurtins, M. \& Cadosch, D. (2010) Recurrent laryngeal nerve monitoring during esophagectomy and mediastinal lymph node dissection. World J. Surg., 34, 2379-2382.

Gockel, I., Kneist, W., Keilmann, A. \& Junginger, T. (2005) Recurrent laryngeal nerve paralysis (RLNP) following esophagectomy for carcinoma. Eur. J. Surg. Oncol., 31, 277-281.

Graboyes, E.M., Bradley, J.P., Meyers, B.F. \& Nussenbaum, B. (2011) Efficacy and safety of acute injection laryngoplasty for vocal cord paralysis following thoracic surgery. Laryngoscope, 121, 2406-2410.

Hulscher, J.B.F., van Sandick, J.W., Devriese, P.P., van Lanschot, J.J.B. \& Obertop, H. (1999) Vocal cord paralysis after subtotal oesophagectomy. Br. J. Surg., 86, 1583-1587.

Igaki, H., Tachimori, Y. \& Kato, H. (2004) Improved survival for patients with upper and/or middle mediastinal lymph node metastasis of squamous cell carcinoma of the lower thoracic esophagus treated with 3-field dissection. Ann. Surg., 239, 483-490.

Iida, T., Tohara, H., Wada, S., Nakane, A., Sanpei, R. \& Ueda, K. (2013) Aging decreases the strength of suprahyoid muscles involved in swallowing movements. Tohoku J. Exp. Med., 231, 223-228.

International Union Against Cancer (UICC) (2009) TNM classification of malignant tumors 7th ed., edited by Sobin, L.H., Gospodarowicz, M.K. \& Wittekind, C. Wiley-Blackwell, New York, NY.

Isshiki, N., Taira, T., Kojima, H. \& Shoji, K. (1989) Recent modifications in thyroplasty type I. Ann. Otol. Rhinol. Laryngol., 98, 777-779.

Isshiki, N., Tanabe, M. \& Sawada, M. (1978) Arytenoid adduction for unilateral vocal cord paralysis. Arch. Otolaryngol., 104, $555-558$.

Jeannon, J.P., Orabi, A.A., Bruch, G.A., Abdalsalam, H.A. \& Simo, R. (2009) Diagnosis of recurrent laryngeal nerve palsy after thyroidectomy: a systematic review. Int. J. Clin. Pract., 63, 624-629.

Jemal, A., Bray, F., Center, M.M., Ferlay, J., Ward, E. \& Forman, D. (2011) Global cancer statistics. CA Cancer J. Clin., 61, 69-90.

Koyanagi, K., Ozawa, S. \& Tachimori, Y. (2015) Minimally invasive esophagectomy performed with the patient in a prone position: a systematic review. Surg. Today, [Epub ahead of print].

Lu, F.L., Casiano, R.R., Lundy, D.S. \& Xue, J.W. (1996) Longitudinal evaluation of vocal function after thyroplasty type I in the treatment of unilateral vocal paralysis. Laryngoscope, 106, 573-577.

McCulloch, T.M., Andrews, B.T., Hoffman, H.T., Graham, S.M., Karnell, M.P. \& Minnick, C. (2002) Long-term follow-up of fat injection laryngoplasty for unilateral vocal cord paralysis. Laryngoscope, 112, 1235-1238.

Miyauchi, A., Inoue, H., Tomoda, C., Fukushima, M., Kihara, M., Higashiyama, T., Takamura, Y., Ito, Y., Kobayashi, K. \& Miya, A. (2009) Improvement in phonation after reconstruction of the recurrent laryngeal nerve in patients with thyroid cancer invading the nerve. Surgery, 146, 1056-1062.

Parkin, D.M., Pisani, P. \& Ferlay, J. (1999) Global cancer statistics. CA Cancer J. Clin., 49, 33-64.

Perie, S., Laccourreye, O., Bou-Malhab, F. \& Brasnu, D. (1998) Aspiration in unilateral recurrent laryngeal nerve paralysis after surgery. Am. J. Otolaryngol., 19, 18-23.

Pertl, L., Zacherl, J., Mancusi, G., Gächter, J.N., Asari, R., Schoppmann, S., Bigenzahn, W. \& Schneider-Stickler, B. (2011) High risk of unilateral recurrent laryngeal nerve paralysis after esophagectomy using cervical anastomosis. Eur. Arch. Otorhinolaryngol., 268, 1605-1610.

Rino, Y., Yukawa, N., Sato, T., Yamamoto, N., Tamagawa, H., Hasegawa, S., Oshima, T., Yoshikawa, T., Masuda, M. \& Imada, T. (2014) Using NU-KNIT ${ }^{\circledR}$ for hemostasis around recurrent laryngeal nerve during transthoracic esophagectomy with lymphadenectomy for esophageal cancer. BMC Res. Notes, 7, 127.

Saikawa, D., Okushiba, S., Kawata, M., Okubo, T., Kitashiro, S., Kawarada, Y., Suzuki, Y. \& Kato, H. (2014) Efficacy and safety of artificial pneumothorax under two-lung ventilation in thoracoscopic esophagectomy for esophageal cancer in the prone position. Gen. Thorac. Cardiovasc. Surg., 62, 163-170.

Schneider, B., Bigenzahn, W., End, A., Denk, D.M. \& Klepetko, W. (2003) External vocal fold medialization in patients with recurrent nerve paralysis following cardiothoracic surgery. Eur. J. Cardiothorac. Surg., 23, 477-483.

Tachimori, Y., Kato, H., Watanabe, H., Ishikawa, T. \& Yamaguchi, H. (1995) Vocal cord paralysis in patients with thoracic 
esophageal carcinoma. J. Surg. Oncol., 59, 230-232.

Tateda, M., Hasegawa, J., Sagai, S., Nakanome, A., Katagiri, K., Ishida, E., Kanno, R., Hasegawa, T. \& Kobayashi, T. (2008) Nonrecurrent inferior laryngeal nerve without vascular anomaly as a genuine entity. Tohoku J. Exp. Med., 216, 133-137.

Walther, B., Johansson, J., Johnsson, F., von Holstein, C.S. \& Zilling, T. (2003) Cervical or thoracic anastomosis after esophageal resection and gastric tube reconstruction: a prospective randomized trial comparing sutured neck anastomosis with stapled intrathoracic anastomosis. Ann. Surg., 238, 803-814.

Zhong, D., Zhou, Y., Li, Y., Wang, Y., Zhou, W., Cheng, Q., Chen, L., Zhao, J., Li, X. \& Yan, X. (2014) Intraoperative recurrent laryngeal nerve monitoring: a useful method for patients with esophageal cancer. Dis. Esophagus, 27, 444-451. 